\title{
Polyposis deserves a perfect physical examination for final diagnosis: Bannayan-Riley-Ruvalcaba syndrome
}

\author{
Hayriye Hızarcıoğlu-Gülşen¹, Esra Kılıç², Elena Dominguez-Garrido ${ }^{3}$, Yusuf Aydemir ${ }^{1}$, \\ Gülen Eda Utine ${ }^{2}$, İnci Nur Saltık-Temizel ${ }^{1}$ \\ Divisions of Pediatric Gastroenterology ${ }^{1}$ and Pediatric Genetics ${ }^{2}$, Department of Pediatrics, Hacettepe University, Faculty \\ of Medicine, Ankara, Turkey, and ${ }^{3}$ Unidad de Diagnostico Molecular, Fundacion Rioja Salud, La Rioja, Spain. \\ E-mail: korkmazkilic@yahoo.com.tr \\ Received: 13th July 2016, Revised: 8th November 2016, Accepted: 26th December 2016
}

SUMMARY: Hızarcıŏlu-Gülşen H, Kılıç E, Dominguez-Garrido E, Aydemir Y, Utine GE, Saltık-Temizel İN. Polyposis deserves a perfect physical examination for final diagnosis: Bannayan-Riley-Ruvalcaba syndrome. Turk J Pediatr 2017; 59: 80-83.

Bannayan-Riley-Ruvalcaba syndrome (BRRS) is a rare autosomal dominant inherited polyposis syndrome characterized by macrocephaly, lipomatosis, hemangiomatosis, intestinal polyposis and pigmented macules on penis. The mutation of the PTEN gene that is responsible for controlling cellular proliferation, migration and apoptosis clarifies the reason of tissue overgrowth in BRRS. Gastrointestinal tract involvement is seen $35-45 \%$ of the patients. Histologic features of polyps in BRRS resemble juvenile polyps. In this report, we describe a boy presenting with hematochezia and aggressive polyposis and finally was diagnosed as BRRS due to extra intestinal findings.

Key words: Bannayan-Riley-Ruvalcaba syndrome, PTEN, polyposis.

Hamartomatous polyps are benign tumoral lesions that are characterized by disorganized overgrowth of normal tissues ${ }^{1,2}$. The hamartomatous polyposis syndromes are a heterogeneous group of disorders including juvenile polyposis syndrome, PTEN hamartoma tumor syndromes; Cowden syndrome (CS), Bannayan-Riley-Ruvalcaba syndrome (BRRS) and Proteus syndrome, Peutz-Jeghers syndrome, multiple endocrine neoplasia syndrome $2 \mathrm{~B}$, hereditary mixed polyposis syndrome, Cronkhite-Canada syndrome, basal cell nevus syndrome and neurofibromatosis type $\mathrm{I}^{2,3}$.

Bannayan-Riley-Ruvalcaba syndrome (OMIM\#153480) is an autosomal dominant inherited hamartomatous polyposis syndrome. The terminology of 'BRRS' was firstly expressed in 1992 to describe three phenotypically similar but previously different denominated syndromes ${ }^{3,4}$. Hallmark manifestations of BRRS are macrocephaly, developmental delay or intellectual disability, subcutaneous and visceral lipomatosis, hemangiomatosis, gastrointestinal polyposis and pigmented macules on glans penis/genitalia ${ }^{3-5}$. Herein, a patient with gastrointestinal polyposis who had a genetically confirmed diagnosis of BRRS is presented.

\section{Case Report}

A 6-year-old boy, born from a nonconsanguineous marriage, was referred to our hospital with complaints of hematochezia and abdominal pain that began nearly 18 months ago. His past medical history showed that he had got a diagnosis of epilepsy 3 years ago and he remained in remission with sodium valproate. He has been both medication and seizure-free for nearly a year. He was macrosomic at birth $(4,000 \mathrm{~g}$ at term) but no complication occurred during perinatal period. He had developmental delay; he achieved head control at 10 months of age, he could sit without support at 18 months of age, and he was able to walk and talk after 2 years of age and at 4.5 years of age, respectively. He was operated for pes equinovarus deformity when he was 1-year-old. His family history was unremarkable.

On physical examination, weight was $18 \mathrm{~kg}$ (25 th percentile), height was $109 \mathrm{~cm}\left(25^{\text {th }}\right.$ 
percentile) and head circumference was $55 \mathrm{~cm}$ $\left(>97^{\text {th }}\right.$ percentile). He had macrocephaly with a prominent and high forehead, hypertelorism, low-set and small ears, micrognathia and high-arched palate, widely-spaced and inverted nipples, café au lait spots on trunk, hyperpigmented macular lesions on glans penis hyperextensible joints, enlarged halluces and thumbs with convex nails, kyphosis, and sandal gap on dysmorphological examination (Fig. 1). Oral mucosa and tongue were normal and he had no trichilemmomas and other cutaneous hamartomas. Denver developmental screening test proved motor and language development delay.

Esophagogastroduodenoscopy and colonoscopy were performed to find out a cause for hematochezia. Three polyps in the 2 nd part of the duodenum and two polyps in the stomach were seen at upper endoscopy, however, colonoscopy revealed innumerable, multilobulated, ulcerated and hemorrhagic polyps with a diameter of 1 to $4 \mathrm{~cm}$ throughout the colon and terminal ileum (Fig. 2). Some parts of the colon lumen were markedly narrowed because of huge polyps. Histological assessment of colonic and duodenal polyps was consistent with juvenile polyps. Barium study supported the presence of duodenal and jejunal polyps.

DNA analysis was performed for BannayanRiley-Ruvalcaba syndrome, as suggested by intestinal juvenile polyposis and accompanying dysmorphic features. Sequence analysis of all exons of phosphatase and tensine homologue (PTEN) gene and multiplex ligation-dependent probe amplification (MLPA) revealed a heterozygous de novo complete deletion of PTEN gene, confirming the clinical diagnosis.

Multiplex ligation-dependent probe amplification was carried out according to the manufacturer's instructions with the P225 PTEN kit (MRC, Holland $\left.{ }^{\circledR}\right)$. The MLPA PCR products were separated by electrophoresis on an ABI3130 Sequencer and MLPA data were evaluated using Coffalyser, a Microsoft Excel based program freely available at the MRC-Holland website.

Informed consent was received from the family.

\section{Discussion}

Bannayan-Riley-Ruvalcaba syndrome is among the rarely encountered and autosomal dominantly inherited hamartomatous polyposis syndromes. This syndrome may be diagnosed in patients with accompanying intellectual disability, macrocephaly and penile pigmentary spotting ${ }^{3}$, as seen in the present patient. Recognizing this combination of clinical features leads to precise genetic diagnosis and genetic counseling.

Although there is still no consensus criterion for diagnosis of BRRS, previously two reports in the literature defined a few diagnostic criteria. Marsh et al. ${ }^{6}$ underlined that patients with BRRS have features of macrocephaly

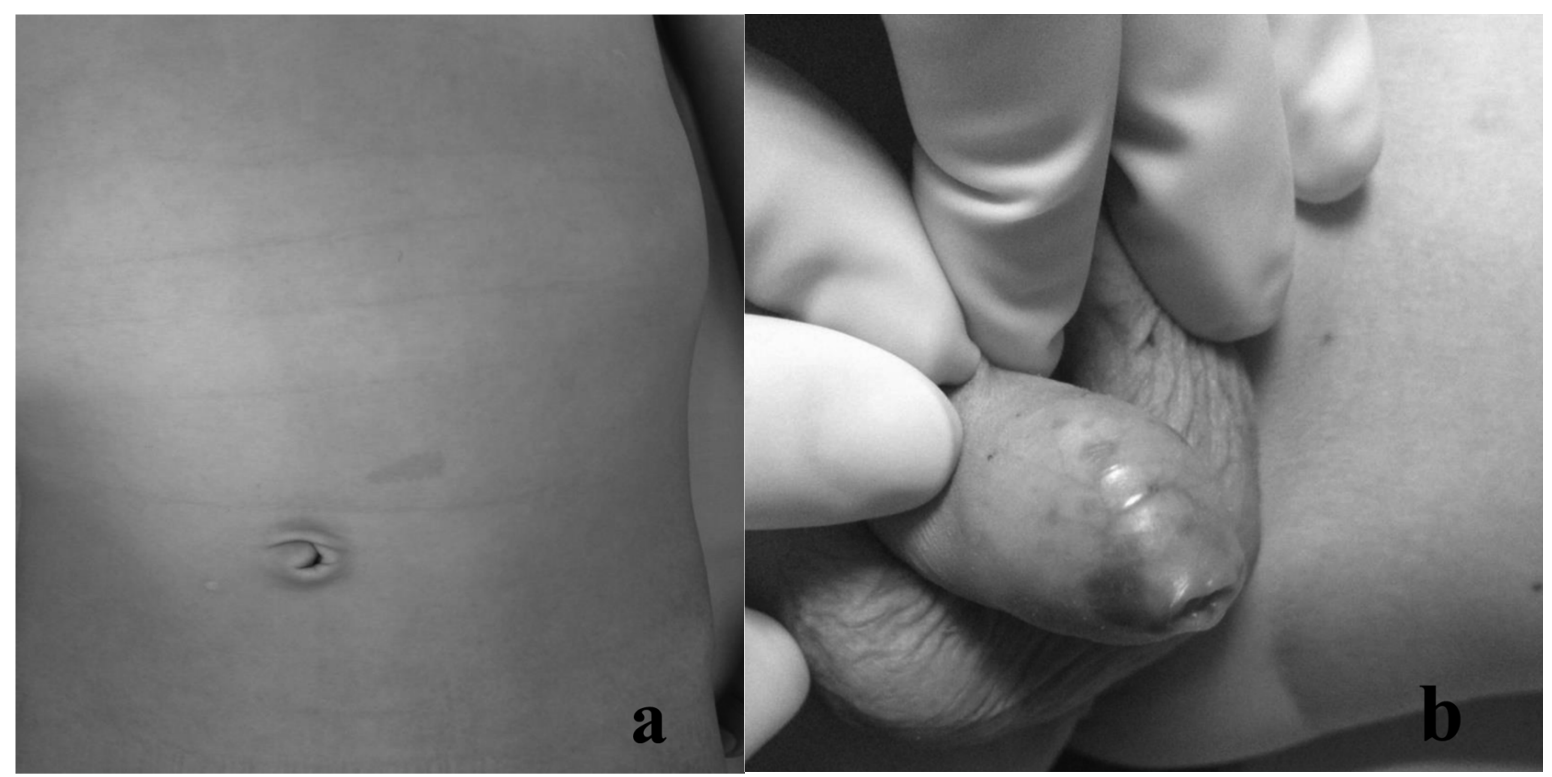

Fig. 1. Café au lait spots (a) and hyper pigmented macular lesions on glans penis (b) 


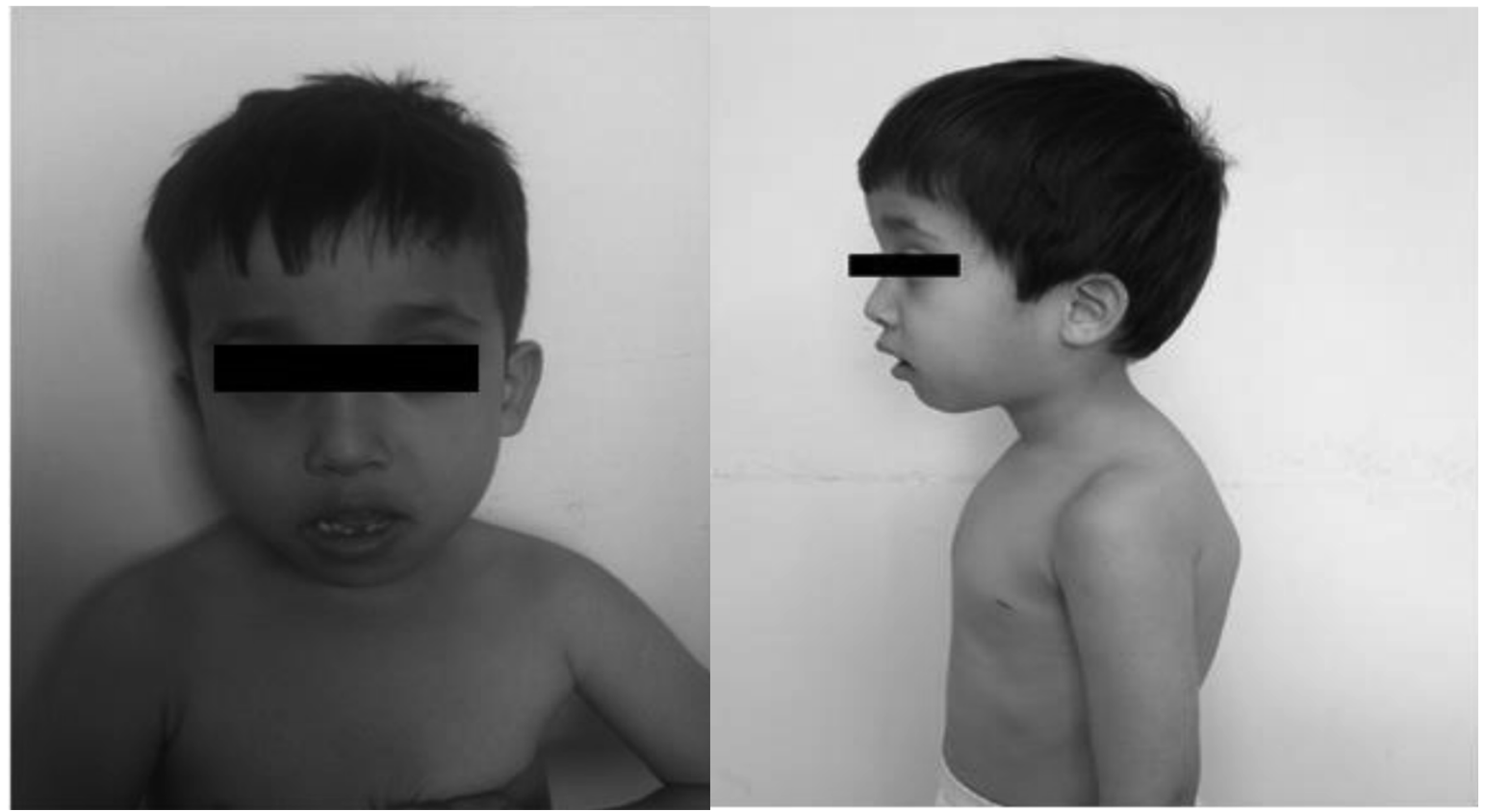

Fig. 2. Dysmorphic features of the patient

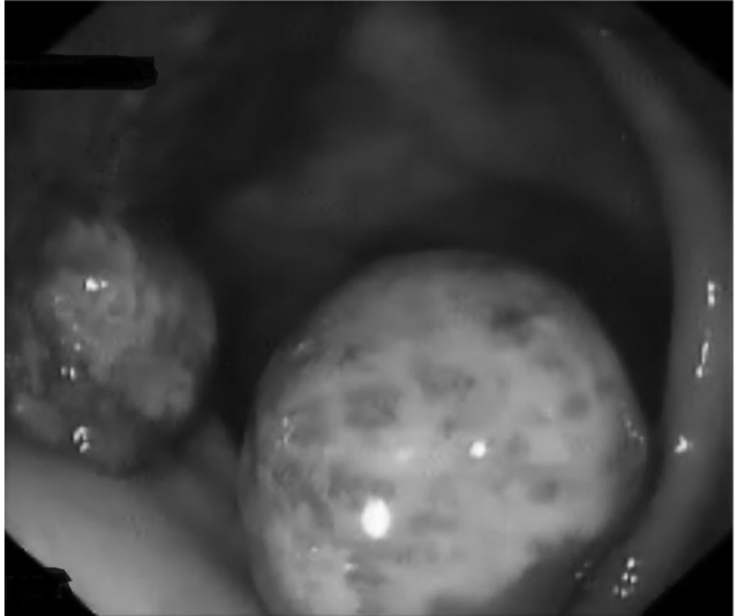

Fig. 3. Polyps in the colon

without ventricular dilatation, lipomatosis and speckled penis. In 2001, Parisi et al. ${ }^{7}$ pointed out that two out of three following criteria were adequate for diagnosis: macrocephaly, hamartomas (lipoma/hemangioma or intestinal polyp) and macules on penis. A recent review article reported that in cases of one or more extra intestinal findings even not presenting with intestinal polyps or family history, BRRS or CS should be considered ${ }^{3}$.

The patients are usually born macrosomic, but late growth rate may decelerate. Hypotonicity with a gross motor delay and mild to severe intellectual disability are common ${ }^{8}$. Accompanying features may also include frontal bossing, down slanting palpebral fissures, pseudo papilledema, high arched palate, long philtrum, small beaked nose, tongue polyps, café au lait spots, pectus excavatum, joint hyper extensibility, scoliosis, pes planus, macrodactyly (accelerated growth of first metacarpal and first proximal and second middle phalanges), hypotonia associated with lipid myopathy, peripheral neuropathy, epilepsy and Hashimoto thyroiditis $3,5,9$. Epilepsy was reported in approximately $25 \%$ of patients ${ }^{8,10}$. After multiple polyps were shown in colonoscopy, extra intestinal features of the present patient raised suspicion about inherited polyposis syndromes, particularly BRRS, and genetic analysis of the PTEN gene confirmed the diagnosis.

Genetic testing is required particularly when a full-blown recognizable clinical picture is absent or overlapping features are observed as there are clinical similarities and atypical presentations of polyposis syndromes. Marsh et al. ${ }^{6}$ reported that approximately $60 \%$ of patients with BRRS are reported to have mutations in PTEN gene, which functions as a tumor suppressor gene and is located on chromosome 10q22-q234-6. Mutations in 
PTEN gene, controlling cellular proliferation, migration, and apoptosis, result in overgrowth syndrome and malignity (PTEN hamartoma tumor syndromes) in BRRS together with allelic CS. BRRS and CS have been insisted to be a single disorder with variability in phenotypic expression and age related penetrance. ${ }^{5}$

Histologic features of polyps in BRRS resemble juvenile polyps ${ }^{1,3}$. Gastrointestinal tract involvement was reported in $35-45 \%$ of the patients $s^{3,11,12}$. Polyps may be seen throughout the gastrointestinal tract but mostly in the distal ileum and colon ${ }^{8}$. Gastrointestinal polyps in BRRS are frequently symptomatic and may present with anemia, chronic diarrhea and small bowel invagination ${ }^{4,11}$. Benign tumors of BRRS may grow rapidly and tend not to show spontaneous resolution ${ }^{12}$. Hence, patients presenting with juvenile polyposis should be re-evaluated for extra intestinal manifestations of BRRS and $\mathrm{CS}^{3}$.

Cancer screening in patients with BRRS is still not clear due to its rarity. A study about genotype-phenotype correlation of PTEN gene revealed that truncating or point mutations were associated with development of any cancer or breast fibroadenoma in BRRS ${ }^{13}$. Hence, a cancer screening program was scheduled for our patient as he had a complete deletion of the gene and increased risk for cancer development. Screening program should include breast, thyroid, endometrial, skin and renal cancers ${ }^{4,5,9}$. As the risk of colon cancer development has not been defined yet, colonoscopy is accepted as a suggestion rather than a part of a definite surveillance program. Baseline screening colonoscopy for the patients with no gastrointestinal symptoms is recommended by at least age 40 and regularly after then at some centers further investigations are needed about colon cancer risks ${ }^{4,8}$.

In conclusion, patients with hamartomatous polyposis should be evaluated carefully with their extra intestinal findings and family history should be questioned in search for probable inherited polyposis syndromes. Precise genetic counseling in patients with gastrointestinal juvenile polyposis requires exact genetic diagnosis and correct genetic testing.

\section{REFERENCES}

1. Lanza G, Messerini L, Gafa R, Risio M. Colorectal tumors: the histology report. Dig Liver Dis 2011; 43 S4: 344-355.

2. Stojcev Z, Borun P, Hermann J, et al. Hamartomatous polyposis syndromes. Hered Cancer Clin Pract 2013; 11: 4.

3. Manfredi M. Hereditary hamartomatous polyposis syndromes: understanding the disease risks as children reach adulthood. Gastroenterol Hepatol (NY) 2010; 6: 185-196.

4. Gammon A, Jasperson K, Kohlmann W, Burt RW. Hamartomatous polyposis syndromes. Best Pract Res Clin Gastroenterol 2009; 23: 219-231.

5. Blumenthal GM, Dennis PA. PTEN hamartoma tumor syndromes. Eur J Hum Genet 2008; 16: 1289-1300.

6. Marsh DJ, Coulon V, Lunetta KL, et al. Mutation spectrum and genotype-phenotype analyses in Cowden disease and Bannayan-Zonana syndrome, two hamartoma syndromes with germline PTEN mutation. Hum Mol Genet 1998; 7: 507-515.

7. Parisi MA, Dinulos MB, Leppig KA, Sybert VP, Eng C, Hudgins L. The spectrum and evolution of phenotypic findings in PTEN mutation positive cases of BannayanRiley-Ruvalcaba syndrome. J Med Genet 2001; 38: 52-58.

8. Edmondson AC, Kalish JM. Overgrowth Syndromes. J Pediatr Genet 2015; 4: 136-143.

9. Piccione M, Fragapane T, Antona V, Giachino D, Cupido F, Corsello G. PTEN hamartoma tumor syndromes in childhood: description of two cases and a proposal for follow-up protocol. Am J Med Genet A 2013; 161A: 2902-2908.

10. Bhargava R, Au Yong KJ, Leonard N. Bannayan-RileyRuvalcaba syndrome: MRI neuroimaging features in a series of 7 patients. AJNR Am J Neuroradiol 2014; 35: 402-406.

11. Poropat F, Maschio M, Martelossi S, Ventura A, Taddio A. Bannayan-Riley-Ruvalcaba syndrome (BRRS): An uncommon case of haematochezia in a 3-year-old-boy. J Pediatr Gastroenterol Nutr 2015; 60: e48.

12. Latiff ZA, Atmawidjaja RW, RajaLope RJ, Syed Omar SA, Syed Zakaria SZ, Jamal RA. Bannayan Riley Ruvalcaba syndrome. Ann Acad Med Singapore 2010; 39: $578-572$.

13. Marsh DJ, Kum JB, Lunetta KL, et al. PTEN mutation spectrum and genotype-phenotype correlations in Bannayan- Riley- Ruvalcaba syndrome suggest a single entity with Cowden syndrome. Hum Mol Genet 1999; 8: 1461-1472. 